\title{
Uso de la ecografía en tiempo estático para la punción lumbar
}

\author{
Use of static-time ecography for lumbar puncture
}

Karla Pamela Romero Ledezma ${ }^{1}$

\section{Resumen}

La ecografía es una herramienta de gran ayuda en medicina, rápido, tecnología portátil y segura. Así por ejemplo realización de la punción lumbar (accesos vasculares y bloqueos regionales) para la obtención de líquido cefalorraquídeo por médicos de emergencia y anestesiólogos, etc. Como ventajas tenemos: disminución del número de punciones lumbares, satisfacción del paciente. Es una alternativa en pacientes con alta probabilidad de punción lumbar difícil como por ejemplo: pacientes obesos, pacientes con edema, con antecedentes de cirugía columna previa, escoliosis. En el presente artículo tiene como objetivo brindar información sobre la utilidad de la ecografía en la punción lumbar en adultos y la obtención del líquido cefalorraquídeo. Proponer una abordaje metódico para puncion lumbar y el bloqueo neuroaxial en tiempo estático por ecografía de la columna lumbar del adulto. Se realizó la búsqueda de la bibliografía de revistas académicas de medicina y anestesiología disponibles en base de datos confiables.

Palabras clave: anestesia, lumbar, ecografia.

\section{Abstract}

Ultrasound is a tool of great help in medicine, fast, portable and safe technology. Thus, for example, the performance of lumbar punctures (vascular accesses and regional blocks) to obtain cerebrospinal fluid by emergency physicians and anesthesiologists, etc. As advantages we have: decrease in the number of lumbar punctures, patient satisfaction. It is an alternative in patients with a high probability of difficult lumbar puncture such as: obese patients, patients with edema, with a history of previous spinal surgery, scoliosis. The aim of this article is to provide information on the usefulness of ultrasound in lumbar puncture in adults and obtaining cerebrospinal fluid. To propose a methodical approach for lumbar puncture and neuraxial block in static time by ultrasound of the lumbar spine in adults. A search was made of the bibliography of academic journals of medicine and anesthesiology available in reliable databases.

Keywords: anesthesia, lumbar, ultrasound.

El uso de la ecografía fue realizada como primera punción según la literatura Rusa en 1971 donde se delineo la anatomía neuroaxial, siendo identificadas como por ejemplo: estructuras de la lámina, ligamento amarillo, canal espinal y el cuerpo vertebral. Así también como medir el espacio de la lámina y el espacio epidural antes del procedimiento epidural ${ }^{1,2}$.

Por otro lado este tipo de utilidad y acceso a través de la ecografía no solo está limitado a los anestesiólogos, sino que también a personal de salud médico para la obtención de la muestra de líquido cefalorraquídeo como los médicos de urgencias, Médicos internistas y Terapia intensiva.

La ecografía está limitada por algunos aspectos que podrían ser la destreza del que realiza el procedimiento, tipo de ecógrafo, tipos de pacientes e interferencias en la imagen. Pero aun así la ecografía es un método más que contribuiría a obtener una imagen que solo es posible a través de los espacios inter-laminares entre vertebras adyacentes donde permitiría el ingreso de las ondas sonoras al canal vertebral y de esta manera también ingresar al espacio con la aguja espinal ${ }^{1}$.

El tipo de búsqueda de información del presente tema de revisión es de contenido explicativo, se realizó la búsqueda

${ }^{1}$ Medico Anestesióloga. Hospital Obrero N² Caja Nacional de Salud. https://orcid.org/0000-0002-7198-5308

${ }^{*}$ Correspondencia a:Karla Pamela Romero Ledezma

Correo electrónico: karla.pameita@hotmail.com

Recibido el 01 de octubre de 2020. Aceptado el 01 de diciembre de 2020. de fuentes primarias y secundarias, de bases de datos reconocidos revistas científicas. Se seleccionó artículos que estén relacionados con el uso de la ecografía para anestesia neuroaxial en pacientes adultos. La validez de los artículos es que estas revistas científicas cumplen con los criterios internacionales en la publicación de artículos científicos como requisitos de uniformidad del Comité internacional de Editores de Revistas Médicas (IMCJE por sus siglas en Ingles).

Se planteó como objetivo de este tema de revisión articulo realizar una revisión descriptiva de la literatura que nos permita brindar la información, sobre la utilidad de la ecografía en la punción lumbar en adultos, la obtención del líquido cefalorraquídeo, procedimiento para la anestesia espinal. Proponer una abordaje metódico para el bloqueo neuroaxial en tiempo estático por ecografía de la columna lumbar del adulto.

\section{Revisión bibliográfica \\ Metodología}

Se realizó una búsqueda bibliográfica en octubre de 2020 en Bases de datos de revistas científicas, utilizando los descriptores: Ecografía and anestesia neuroaxial, artículos de revisión, artículos originales, resúmenes de artículos en proceso de elaboración publicación cortas. Se obtuvo como 20 de 117 registros tras combinaciones las palabras claves en "Anesthesiology" revista norteamericana reconocida 
internacionalmente. Además se realizó la búsqueda en internet en el buscador "google académico" con términos similares. Teniendo como criterios de selección de los artículos como: uso de la ecografía en anestesia neuroaxial, anestesia espinal, procedimientos para realizar la el bloqueo neuroaxial guiado por ecografía en pacientes adultos.

\section{Desarrollo}

Anatomía de la columna: De forma típica la vértebra está formada por: un cuerpo y arco. Así este último tendría las siguientes partes como ser: pedículo, lamina, apófisis espinosas y articulares superior e inferior. Así se tiene que entender algunos términos que serían "espacio interlaminar" sinónimo de las láminas adyacentes y "espacio interespinoso" sinónimo de apófisis espinosas en la descripción de este escrito.

Se observó que las únicas ventanas acústicas que tendremos que buscar será: agujeros intervertebrales (lateral) y espacios inter-laminares (posterior). Ligamento flavum es el que une los espacios inter-laminares. El ligamento amarillo teniendo un componente superficial y profundo. Y las apófisis espinosas unidas por el ligamento interespinoso. El canal vertebral que está formada por saco tecal mismo que contiene duramadre y aracnoides.

El ligamento flavum desde 1995 se lo reconoció ecográficamente pudiendo ser de ayuda para identificar y acceder al espacio epidural. Acompañado con el uso de transductor curvo con frecuencias bajas de $4-7 \mathrm{MHz}^{3}$.

También está formada por cinco vertebral lumbares teniendo una dirección anterosuperior, y torácicas teniendo apófisis espinosa anchas y planas casi verticales.

El cono medular generalmente en el adulto se encuentra en L1, primera lumbar, y el saco tecal termina a nivel de la primera sacra $S 1^{1}$.

Por otro lado entender algunos aspectos, principios de la ecografía es importante como ser: los tipos de transductores y sus usos de cada uno de ellos evitara que se realice el uso del inadecuado para las diferentes estructuras a inspeccionar, términos ecográficos como ser: anecoico la cual está representado de color negro indica una estructura que no genera ecos, hipoecoico de color gris la cual indica que genera ecos de poco o baja intensidad. E hiperecoico de color blanco brillante la que genera ecos de gran intensidad. Tipos de corte que pueden ser transversal, sagital, etc ${ }^{4}$.

Orientación de la imagen la relacionada con un lado de la pantalla, es como si estuvieses observándolo al paciente en vista de frente ${ }^{5}$.

Indicaciones para la realización de uso de la ecografía neuroaxial espinal. La realización de esta habilidad o competencia en el personal de salud y ahora para el anestesiólogo, implica un compromiso de educación continua, con el objetivo de brindar una mejor calidad de atención al paciente y así evitar las complicaciones del paciente. Estaría indicado en aquellas columnas de probable punción difícil teniendo como variables para esta: edad, puntos de referencias no palpables (obesidad, edema), antecedentes personales de cirugía en la columna previa, o columna deformante como espondilitis anquilosante, escoliosis. Siendo una puntuación de igual o mayor a cuatro puntos predeciría punción difícil ${ }^{6,7}$.

Técnica de abordaje ordenado para el bloqueo en tiempo estático por ecográfica dela columna lumbar de adulto para la anestesia neuroaxial espinal.

Puntuación y valoración de paciente para posibles predictores de dificultad de punción lumbar.

1. Se procederá a realizar una explicación al paciente sobre el procedimiento que se realizara previo a su cirugía, también sobre el uso de la ecografía que será descrito a continuación $^{1}$ : la preparación para la exploración, se posicionara al paciente para el procedimiento de boqueo pudiendo ser sentado o decúbito dorsal lateral.

Se recomienda el uso de transductor curvo de baja frecuencia (2-5 Mhz). También se puede utilizar el transductor de alta frecuencia o transductor lineal (10-12 Mhz) que brinda en el monitor una imagen rectangular, tiene baja penetrancia $(2-4 \mathrm{~cm})$ pero con alta resolución de la imagen considerar este transductor en pacientes delgados. Se procederá a realizar el ajuste del ecógrafo: generalmente $7-10 \mathrm{~cm}$, ajuste de enfoque y ganancia en la máquina de ultrasonido según el caso necesario. No debe descuidarse algunos aspectos como ser: reducir la frecuencia del haz, presión adecuada del transductor de la piel que tenga un buen contacto y comprima el tejido suprayacente. Tener lista la aguja número 22 o mayor con longitudes mayores de $90 \mathrm{~mm}$. O también la aguja 25 G B Braun- Alemania, Aguja punta de lápiz.

El transductor debe estar protegido con una aislante previa colocación del gel. Otra opción sería realizar el marcaje con marcador demográfico, luego se retira de forma cuidadosa el material de gel de la piel el marcado en la piel será con marcador indeleble, es importante la adecuada eliminación de restos de gel de la piel del lugar a puncionar porque no se sabe qué complicaciones podría causar, si este gel ingresara al espacio raquídeo. Entonces algunos sugieren que podría hacer el reemplazo del gel por suero fisiológico para evitar este evento ${ }^{7}$.

Se debe tener una técnica aséptica completa con guantes, bata, mascarilla. También listo el material estéril para realizar la punción lumbar.

Considerar la sedación del paciente de acuerdo a las características del mismo, aunque será solo el marcaje con ecógrafo no así una punción en tiempo real con el transductor. Ya que este último tiene una descripción diferente y también es realizado ya por anestesiólogos experimentados. Se brindara al paciente la administración de oxigeno con una mascarilla.

2. Se procede al reconocimiento anatómico con la ayuda del ecógrafo. Observar la apófisis transversa de para mediana sagital, colocar la sonda de transductor en paramediano sagital a $3-4 \mathrm{~cm}$ de la línea media. Entonces el "signo de tridente" observara siendo representado por las sombras acústicas en forma de dedos de los procesos transversales. A nivel de L2-L3 se observa este signo. Recordar que el 


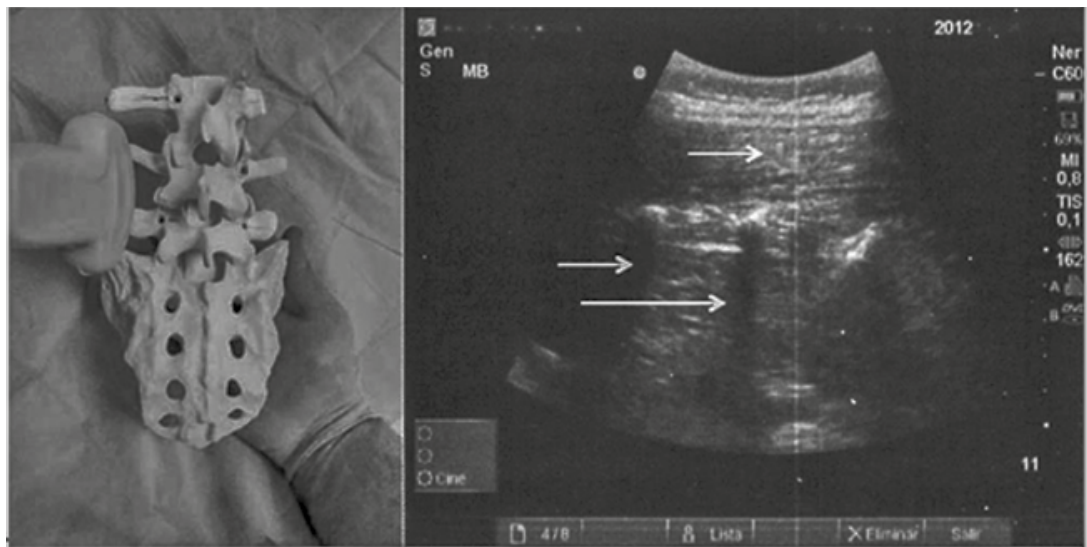

Figura 1. Abordaje longitudinal (sagital) paramedial. Identificación de apófisis transversas

Fuente: Ramirez CR. Anestesia obstétrica y ecografía. Revista Mexicana de Anestesiologia

plexo lumbar se encuentra más de $2 \mathrm{~cm}$ por debajo de la apófisis transversa ${ }^{8,9}$ (Figura 1 ).

3. Observar de la apófisis articular de la paramediana sagital. Colocar la sonda medialmente hacia la línea media manteniendo una orientación, obteniendo la imagen de "jorobas" redondeadas de las articulaciones facetarías entre las apófisis articulares superior e inferior (Figura 2).

4.- Observar con vista oblicua paramediano sagital, buscar la imagen de la apófisis articular paramediana sagital, incline la sonda hacia la línea media para obtener la vista oblicua Paramediano sagital. Es posible que se requieran pequeños movimientos adicionales de deslizamiento e inclinación de la sonda para optimizar la vista. Obtendremos una apariencia de "diente de sierra" de las láminas. A nivel de L2-S1 se observa esa imagen, entonces ver el ligamento longitudinal posterior (PLL) se tendrá que calificar como una buena imagen si fuese brillante (pudiendo también observarse ausente o brumoso), la ausencia de este imagen ecográfica es predictor de punción espinal difícil ${ }^{10}$ (Figura 3 ).
Complejo posterior (ligamento amarillo, espacio epidural y duramadre posterior). Complejo anterior (dura anterior, ligamento longitudinal posterior, cuerpo vertebral) ${ }^{9}$.

Para el conteo vertebral será identificando primero el sacro que ecográficamente se lo observa colocando el transductor sobre el sacro entre $2-3 \mathrm{~cm}$ a la derecha de la línea media así visualizar la línea hiperecogenica correspondiente al sacro. Mover en dirección cefálica obtener la imagen ya mencionada "sierra", "nudillos de mano en puño" que son los procesos articulares pudiendo llegar a L3-L41".

Realizar movimiento lento cefálico a caudal hasta obtener ausencia de sombra acústica. A veces no se puede identificar la imagen del complejo ligamento amarillo y la duramadre como dos estructuras. Solamente podríamos observar una línea hiperecogenica única, entonces marcar con bolígrafo demográfico correspondientes al punto de punción ${ }^{12}$.

5. Identificar y marcar los niveles intervertebrales: deslizar con la sonda movimientos en sentido caudal manteniendo una orientación Paramediana Sagital oblicua, hasta que el espacio intervertebral L5-S1 quede centrado en la pantalla
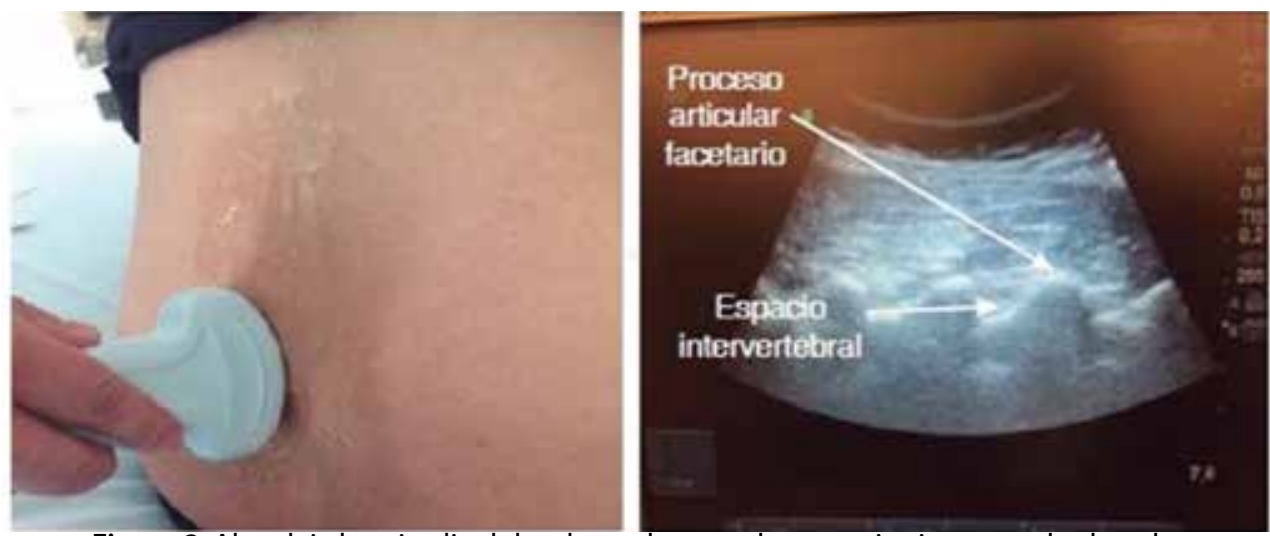

Figura 2. Abordaje longitudinal donde se observan los espacios intervertebrales y la imagen en dientes de serrucho o nudillos de puño de mano.

Fuente: Ultrasonografía del espacio epidural lumbar vol.40, №2, 2017 


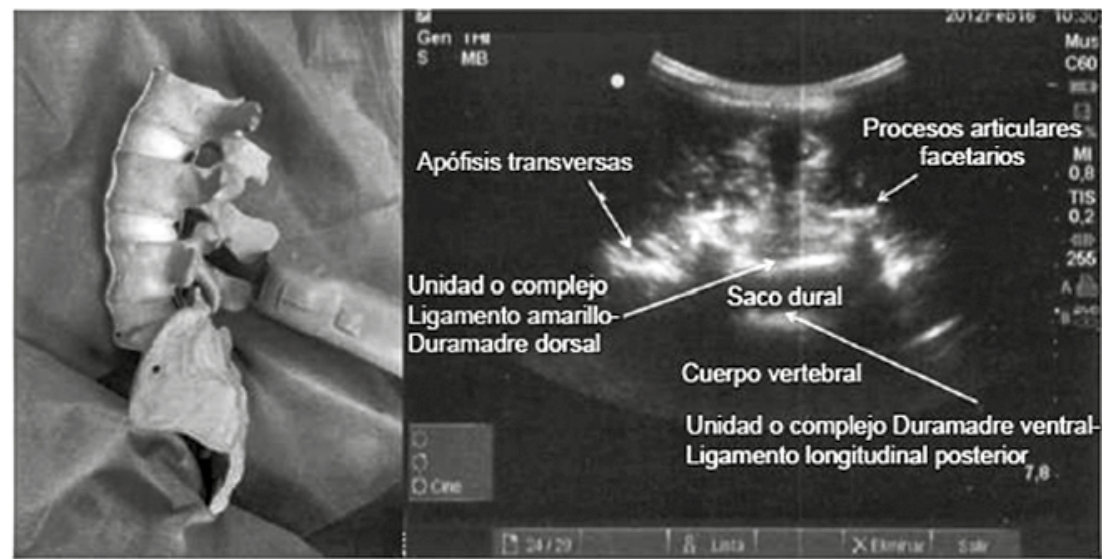

Figura 3. Representación del espacio intervertebral en un abordaje transversal con todos los elementos identificables. Corte que permite medir las distancias entre piel y ligamento amarillo, asi como la continuidad del mismo, imagen de "murciélago volando"

Fuente: Ramirez CR. Anestesia obstétrica y ecografía. Revista Mexicana de Anestesiología

Ultrasonido, su ubicación corresponderá con el punto medio del lado largo de la sonda y puede estar marcado en la piel del paciente.

Posteriormente se moverá la sonda en dirección cefálica, centrando cada espacio intervertebral sucesivo (L4-L5, L3L4, L2-L3) en la pantalla de ultrasonido Y márquelo en la piel del paciente (método de "conteo progresivo" de la porción sacra hacia arriba). La identidad de los espacios intervertebrales puede confirmarse identificando la vértebra T12 por su articulación con la vigésima costilla y luego deslizando la sonda en dirección caudal para visualizar cada espacio intervertebral sucesivo (el enfoque de "cuenta regresiva"). Es importante identificar la línea horizontal hiperecoica del sacro. La vigésima costilla y su articulación con el proceso transversal de la vértebra T12.

6.- Observar transversal interlaminar: gire la sonda 90 grados en orientación transversal y deslícela cefálica o caudalmente según sea necesario para obtener vistas transversales interlaminares de los espacios intermedios deseados (L2-L3, L3-L4, L4-L5). Es permitido que la sonda haya inclinarse en dirección cefálica para optimizar la vista. Calcule la profundidad de inserción de la aguja requerida midiendo la profundidad desde la piel hasta el complejo posterior utilizando los calibradores electrónicos de la máquina de Ultrasonido ahora es esencial ver la apófisis articulares del ligamento interespinoso y apófisis transversas complejo anterior complejo posterior.

La medición será realizada con el botón de medida o caliper del ecógrafo que está integrado en la botonería su función será importante ya que nos ayuda a medir las proporciones de las estructuras que estamos estudiando o las velocidades de la sangre en los estudios vasculares. O puede también estar ubicado el calipre en la pantalla. Entonces medimos la distancia de la piel hasta la porción anterior del complejo ligamento amarillo-duramadre q será el límite inferior de la profundidad intratecal o una aproximación de la profundidad del espacio epidural ${ }^{12,9}$. Esta sería una medición del espacio epidural. Siendo esta una medición indirecta del espacio o profundidad intratecal. Esto en plano o abordaje transversal y el paramediano longitudinal como herramienta complementaria (Figura 4).

7. Ahora se deberá marcar el punto de inserción de la aguja para un abordaje de línea media: centrar la línea media neuroaxial en la pantalla de ultrasonido. En la vista interlaminar transversal y marcar el punto medio de los lados largo y corto de la sonda. La intersección de estas dos marcas indica el punto de inserción de la aguja. Realice la anestesia espinal o epidural de la forma habitual, guiado por las marcas de la piel y las medidas de profundidad. Las redirecciones de la aguja, si es necesario, suelen ser pequeñas y en dirección cefálica.

Algunas consideraciones en pacientes con predictores de punción lumbar difícil como ser: en pacientes de edad avanzada los espacios interespinosos e interlaminares los estrechos. Lo cual dificulta la imagen, entonces con una vista transversal del canal vertebral puede ser difícil su visualización se sugiere realizar vista oblicua paramediano sagital sería una buena opción. El marcaje previo en estos pacientes por tener una la piel flácida y móvil puede variar la punción. Bien para solucionar esta movilidad de la piel, podría ser conveniente la ayuda de una persona para que la piel se mantenga firme donde inicialmente se realizó el marcaje.

La literatura menciona que el espacio interespinoso e interlaminar en el nivel L5-S1 es el más fácilmente localizado según ultrasonido por lo que es de utilidad en el anciano ${ }^{13}$.

En paciente obeso identificar y obtener una imagen con vista parasagital del sacro, después mover la sonda en sentido cefálico para posteriormente identificar las láminas vertebrales lumbares de L5 a L1. Cuando este en un nivel L3-L4 la sonda deberá girar $90^{\circ}$ grados obteniendo una vista transversal, el espacio de apófisis espinosas identificar el complejo Flavo o dura a lo largo de la línea media. Luego marque en la piel esta referencia ${ }^{14,9}$. O en la mujer 

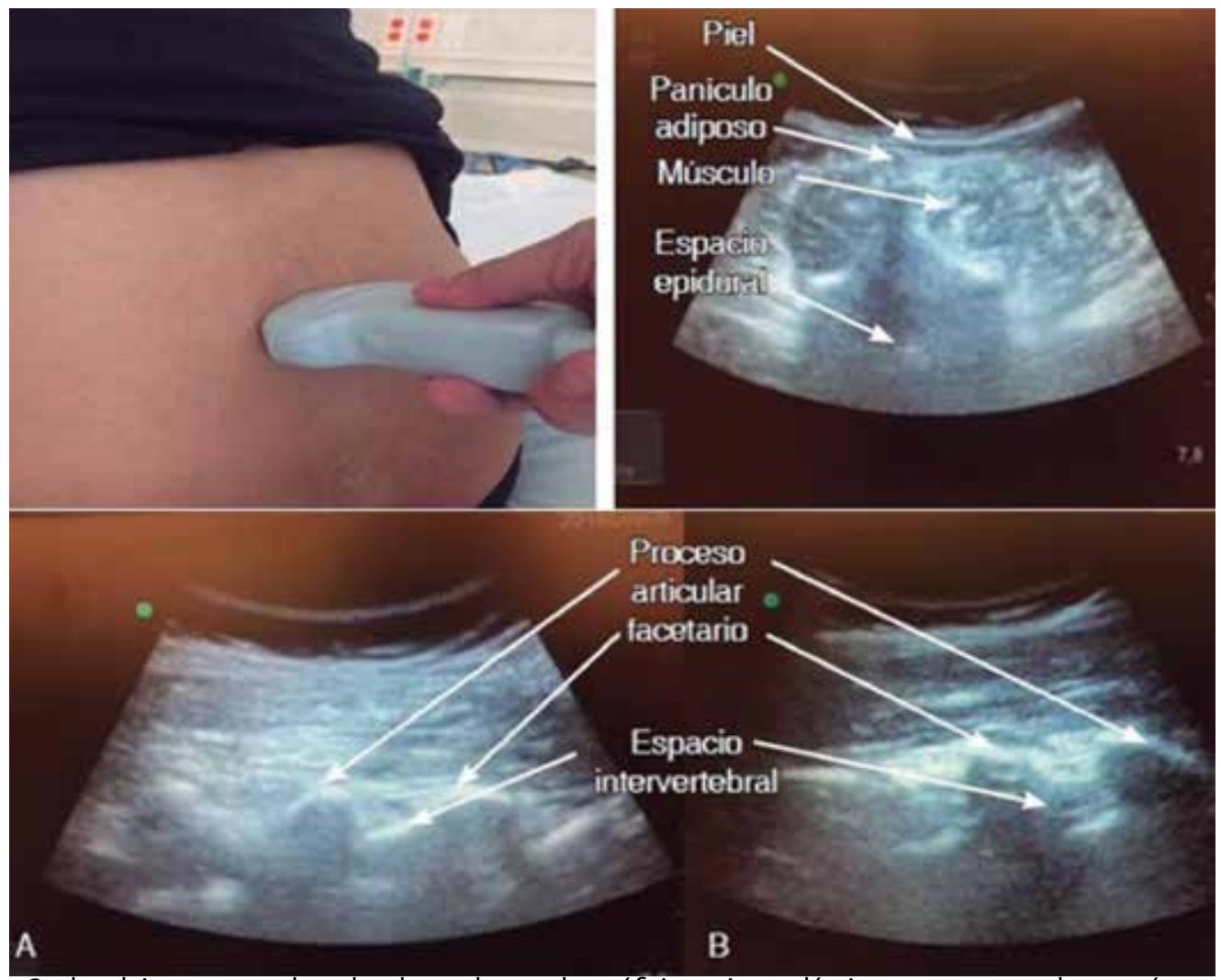

Figura 3. abordaje transversal en donde se observa la apófisis espinosa, láminas, masa muscular común, espacio epidural y canal medular. 7: Imagen a nivel L3-L4 en donde se muestran las diferencias anatómicas ultrasonografías entre dos voluntarios. 7.A Femenino de $1.56 \mathrm{~m}$ e IMC 28 y 7.B masculino de $1.70 \mathrm{~m}$ e IMC 27.

Fuente: Carrillo- Esper R y cols. Ultrasonografía del espacio epidural lumbar Vol.40, №2, 2017

embarazada con retención de líquidos si presenta dificultad en su visualización podría realizar lo siguiente como una presión sobre el transductor así comprimir las estructuras subcutáneas. Aunque variara un poco la distancia estimada pero te ayudara a una mejor visualización.

Ahora paciente con cirugía de columna puede estar la imagen o visualizar la metalistería si hay una vía para poder realizar el bloqueo neuroaxial ${ }^{6}$.

\section{Discusión}

El uso de la ecografía está siendo aplicada en varias áreas de la medicina, es así que en el presente tema de revisión se describe la información de acuerdo a nuestro objetivo que es de brindar información sobre el uso de la ecografía para realizar la identificación del espacio lumbar, teniendo este dato nos permite tener acceso a la obtención de la punción lumbar en el ámbito de Anestesiología, podremos brindar al paciente una anestesia neuroxial espinal. Se ha visto en los estudios que mejora la calidad de atención del paciente y grado de satisfacción del mismo, porque tiene menor número de manipulaciones de la aguja para el éxito y el tiempo necesario para el bloqueo.

$\mathrm{Al}$ mismo tiempo disminuye las posibles complicaciones como ser: si se realiza punción peridural evita la punción inadvertida de la duramadre, punción vascular y parestesia. Estos últimos redujeron hasta el cincuenta por ciento ${ }^{1}$.
También con su uso se puede predecir punción lumbar difícil, dentro de estos criterios se tiene: presencia de deformidad, obesidad (índice de masa corporal mayor 33 $\mathrm{kg} / \mathrm{m}^{2}$ ) antecedente de punción lumbar difícil, cirugía de columna, ausencia de puntos de referencia por palpación, pacientes con edema en región a puncionar ${ }^{1}$.

También estos pacientes con predictores de punción lumbar difícil podrían dificultar en la obtención de la imagen ecográfica brindándonos imágenes de mala calidad. Como por ejemplo: obesos las estructuras están atenuadas que se produce cuando las ondas viajan a una distancia mayor de tejidos blandos ${ }^{15}$. Una posible ayuda para solucionar esta dificultad con la ayuda de la tecnología del ecógrafo podría ayudarnos usar el ecógrafo imágenes armónicas.

Se observó en algunos estudios que para adquirir esta competencia de uso de la ecografía para la punción espinal se requiere tener una práctica de un promedio de 40 pacientes para adquirir esta habilidad o competencia ${ }^{1}$.

La mayoría de los estudios para evaluar la medición de la profundidad por ultrasonido, son para realizar anestesia epidural, siendo como medidas encontradas realizada en 300 pacientes una profundidad promedio de $51,2+/-7 \mathrm{~mm}$ Graul y col. en anestesia epidural y otro estudio realizado en 100 pacientes profundidad de $53+/-7 \mathrm{~mm}$ Grul y col. ${ }^{12}$ por lo que sería recomendable en un futuro no lejano realizar más estudios para realizar comparaciones. 


\section{Conclusiones}

Podemos concluir que el uso de la ecografía es importante como una herramienta de gran utilidad para el beneficio del paciente. Es así que todos los médicos deberían estar capacitados para su uso adecuado cuando así se lo requiera, no estamos exentos los anestesiólogos, deberíamos adquirir esa competencia, la literatura sugiere que primero se tendría que reconocer los conocimientos básicos de ecografía, así mismo lo recomendable seria desde los primeros años de estudio en la residencia, debe ser cultivada esta capacidad o competencia para que en un futuro tener mayor pericia en ese ámbito amplio de la ecografía.

Realizar la practica con pacientes de anatomía normal de la columna para que después avanzar a pacientes con predictores de punción lumbar difícil.
La poca práctica que se tiene con la ecografía es debido al poco acceso de ecógrafo en la cabecera de paciente en servicio de desempeño, que además sea de sofisticado. Lo cual dificulta el reconocimiento de la anatomía espinal ultrasonografía para desarrollar la habilidad intervencionista.

Sin embargo con la experiencia adquirida se podría realizar el procedimiento de anestesia neuroaxial guiada por ultrasonido en tiempo real, también requiere de un persona capacita para que te ayude en el procedimiento.

Entonces el uso de la ecografía en tiempo estático para anestesia espinal, ayuda para brindar una anestesia de calidad porque hay un aumento en la satisfacción del paciente, menos intentos para poder realizar la anestesia espinal, mejora la seguridad en el paciente.

\section{Referencias bibliográficas}

1. Ki Jinn Chin FRCPCMKK, M.D.; Philip Peng, F.R.C.P.C.; David S. Warner, M.D. Ultrasonography of the Adult Thoracic and Lumbar Spine for Central Neuraxial Blockade Anesthesiology [Internet]. 2011 [cited 2020; Vol. 114(6):[145985 pp.]. Available from: https://pubs.asahq. org/anesthesiology/article/114/6/1459/12806/ Ultrasonography-of-the-Adult-Thoracic-andLumba.

2. Esteban QPJ. Ecografía para anestesiólogos2014; vol. 42:[33-6 pp.]. Available from: http://www. scielo.org.co/pdf/rca/v42n1/v42n1a07.pdf.

3. MD A. Ecografia para acceder al canal vertebral: mito o realidad2008; Vol 31:[p 15 p.]. Available from: https://www.medigraphic.com/pdfs/rma/ cma-2008/cmas081b.pdf.

4. Ortega-Romero A D-RT, Concepción delOlmo, Maryem-Fama Ismael, Mayoral, MD V. Ultrasound-guided interventional procedures for lumbar pain2013; Vol. 17:[96-106 pp.]. Available from: http://www.asociacionandaluzadeldolor.es/ wp-content/uploads/2017/02/US-lumbar.pdf.

5. Asenjo J MJ, Fernndez E. Taller analgesia regional continua guiada por ecografia. Revista Boliviana del dolor. 2015; Vol 9:pag. 63-5. español.

6. Mc Donald A MH. Anestesia neuroaxial guiada por Ultrasonido2017; 349:[pag. 1-10 pp.]. Available from: https://www.sbahq.org/wpcontent/uploads/2017/04/349_portugues.pdf.

7. Conroy PH, Luyet C, McCartney CJ, McHardy PG. Real-Time Ultrasound-Guided Spinal Anaesthesia: A Prospective Observational Study of a New Approach. Anesthesiology Research and Practice. 2013 2013/01/10;2013:525818.

8. AsenjoJuan Francisco M, Fernandez Enrique TALLER ANALGESIA REGIONAL CONTINUA GUIADA POR ECOGRAFÍA2015; Vol 9:[68 p.]. Available from: http://www.dolor-bolivia.org.bo/ img/revista9.pdf.

9. Carlos Rafael Ramírez-Paesano ZSH-S, María Gabriela Silva-Hernández ${ }^{*}$. Anestesia neuroaxial guiada por ultrasonografía en la embarazada2012; Vol. 35. :[pp 245-54 pp.]. Available from: https:// www.medigraphic.com/pdfs/rma/cma-2012/ cma124d.pdf.

10. Ramírez $\mathrm{Cl} \mathrm{HZ,} \mathrm{Silva} \mathrm{M.} \mathrm{Anestesia} \mathrm{neuroaxial}$ guiada por ultrasonografía en la embarazada2012; Vol. 35. No. 4 [pp 245-54 pp.]. Available from: https://www.medigraphic.com/pdfs/rma/cma2012/cma124d.pdf.

11. Raúl Carrillo-Esper JADP-M, Gustavo Huesca-Jiménez, Idalia A Ibarias-Enciso etal. Ultrasonografía del espacio epidural lumbar2017; Vol. 40:[pp 134-7 pp.]. Available from: https:// www.medigraphic.com/pdfs/rma/cma-2017/ cma172k.pdf.

12. Gnaho Al NV, Villevielle T, Frota M, etal. Evaluacion de la profundidad del Espacio subaracnoideo con el uso del ultrasonido2012; vol 62:[pp 1-5 pp.]. Available from: https://www. scielo.br/pdf/rba/v62n4/es_v62n4a05.pdf.

13. Liu Fei MD, Li Jia Wei, Ph.D., Branchobporn Songthamwat, M.D., Karmkar Manoj Kumar, M.D. Ultrasound Visibility of the Neuraxial Structures at the L3-4-5 and L5-S1 Intervertebral Level in the Elderly2014; Room Hall B1-Area A:[1 p.]. Available from: http://www.asaabstracts.com/ strands/asaabstracts/abstract.htm?year=2014\&in dex $=17 \&$ absnum $=3790$.

14. Govindarajulu Arun Prasad MD, F.R.C.A., DA, Anahi Perlas, M.D., F.R.C.P.C., Ki Jinn, M.D., F.R.C.P.C., Vincent Chan, M.D., F.R.C.P.C. Ultrasound Assisted Spinal Anesthesia in Morbidly Obese Patients2008; A339:[1 p.]. Available from: http://www.asaabstracts.com/ strands/asaabstracts/abstract.htm?absnum $=1486$ \&index $=17$ \&year $=2008$.

15. R R-M. Opciones de abordaje neuroaxial. Acceso de Taylor2016; Vol. 39:[pp S182-S4 pp.]. Available from: https://www.medigraphic.com/ pdfs/rma/cma-2016/cmas161bj.pdf. 